\title{
Cateterização venosa central de longa permanência em adolescentes com doenças onco-hematológicas
}

\author{
Long-term central venous catheterization in adolescents with onco-hematological diseases \\ Cateterismo venoso central de larga duración en adolescentes con enfermedades oncohematológicas
}

Recebido: 10/04/2021 | Revisado: 16/04/2021 | Aceito: 20/04/2021 | Publicado: 25/04/2021

Juliana Almeida de Oliveira

ORCID: https://orcid.org/0000-0002-7709-6114

Universidade do Estado do Rio de Janeiro, Brasil

E-mail: ju.aoliveira@yahoo.com

Ellen Marcia Peres

ORCID: https://orcid.org/0000-0003-4262-6987

Universidade do Estado do Rio de Janeiro, Brasil

E-mail: ellenperes@globo.com

Helena Ferraz Gomes

ORCID: https://orcid.org/0000-0001-6089-6361

Universidade do Estado do Rio de Janeiro, Brasil

E-mail: helenafg1@yahoo.com.br

Dayana Carvalho Leite

ORCID: https://orcid.org/0000-0001-6354-9111

Universidade do Estado do Rio de Janeiro, Brasil

E-mail: dayanaleite@hotmail.com

Priscila Cristina da Silva Thiengo de Andrade

ORCID: https://orcid.org/0000-0003-0840-4838

Universidade do Estado do Rio de Janeiro, Brasil

E-mail: profprithiengo@gmail.com

Carolina Cabral Pereira da Costa

ORCID: https://orcid.org/0000-0002-1345-0612

Universidade do Estado do Rio de Janeiro, Brasil

E-mail: carolcuerj@hotmail.com

Cristiene Faria

ORCID: https://orcid.org/0000-0001-6548-1851

Universidade do Estado do Rio de Janeiro, Brasil

E-mail: cristienefaria54@gmail.com

Thais Mayerhofer Kubota

ORCID: https://orcid.org/0000-0001-8782-8932

Universidade do Estado do Rio de Janeiro, Brasil

E-mail: thaismk@gmail.com

Harlla Eduarda Santana Torres

ORCID: https://orcid.org/0000-0002-1484-0057

Universidade do Estado do Rio de Janeiro, Brasil

E-mail: harlla.eduarda2016@gmail.com

Iasmin da Silva Barcelos

ORCID: https://orcid.org/0000-0003-2093-7880

Universidade do Estado do Rio de Janeiro, Brasil

E-mail: iasminbarcelos1996@gmail.com

Laura Queiroz dos Anjos

ORCID: https://orcid.org/0000-0003-2759-2379

Universidade do Estado do Rio de Janeiro, Brasil

E-mail: queiroz.laura98@gmail.com

Thelma Teti Toledo

ORCID: https://orcid.org/0000-0002-9364-0730

Universidade do Estado do Rio de Janeiro, Brasil

E-mail: thelmatoledo@gmail.com

Bruna Maiara Ferreira Barreto Pires

ORCID: https://orcid.org/0000-0002-5584-8194

Universidade Federal Fluminense, Brasil

E-mail: bruna.barreto07@gmail.com

\section{Resumo}

Objetivo: analisar o uso dos cateteres venosos centrais, de longa permanência, em adolescentes com doenças oncohematológicas, atendidos no período compreendido entre 2008 e 2018, em uma enfermaria especializada de atenção integral a saúde do adolescente. Método: Estudo descritivo transversal, retrospectivo, de análise documental, com 
abordagem quantitativa, desenvolvido em um Hospital Universitário do Estado do Rio de Janeiro, Brasil. Resultados: Amostra composta por 49 impressos sobre as condições clínicas de adolescentes com doenças onco-hematológicas submetidos à implantação dos cateteres venosos centrais. 65\% $(\mathrm{n}=32)$ eram do sexo masculino com a idade mínima e máxima de 12 e 19 anos, sendo 22,45\% (n=11) com a idade de 15 anos. Quanto às doenças onco-hematológicas, observou-se predomínio das leucemias destacando-se a Leucemia linfoide aguda, representada por 43\% ( $\mathrm{n}=21)$ dos casos, seguida dos linfomas em $35 \%(n=17)$. Dentre os tipos de dispositivos, houve predomínio do Cateter Central de Inserção Periférica em 71,43\% ( $\mathrm{n}=35)$, e dentre os motivos de retirada dos cateteres prevaleceu a ausência de registros em $51,02 \%(n=25)$, seguida da obstrução e da infecção, ambas com 12,24\% (n=6). Em relação ao tempo de permanência, notou-se que dos 49 cateteres implantados, 55,10\% $(n=27)$ apresentou ausência dos registros e 36,74\% (n=18) permaneceram em um tempo menor que um ano. Conclusão: Os dados evidenciados reforçam a necessidade dos registros de enfermagem e da aplicação de boas práticas de manutenção e manuseio destes cateteres. Ressalte-se a importância da capacitação da equipe de enfermagem, a fim de promover a segurança do paciente por meio de uma assistência qualificada de enfermagem.

Palavras-chave: Enfermagem; Saúde do adolescente; Neoplasia; Hematologia; Cateterismo venoso central.

\begin{abstract}
Objective: to analyze the use of long-term central venous catheters in adolescents with onco-hematological diseases, treated in the period between 2008 and 2018, in a specialized ward of comprehensive care for adolescent health. Method: Cross-sectional, retrospective, documentary analysis study with a quantitative approach, developed at a University Hospital in the State of Rio de Janeiro, Brazil. Results: Sample consisting of 49 forms on the clinical conditions of adolescents with onco-hematological diseases submitted to the implantation of central venous catheters. $65 \%(\mathrm{n}=32)$ were male with a minimum and maximum age of 12 and 19 years old, with $22.45 \%(\mathrm{n}=11)$ aged 15 years. As for oncohematological diseases, leukemias predominated, especially acute lymphoid leukemia, represented by $43 \%(\mathrm{n}=21)$ of the cases, followed by lymphomas in $35 \%(\mathrm{n}=17)$. Among the types of devices, there was a predominance of the Peripherally Inserted Central Catheter in $71.43 \%(\mathrm{n}=35)$, and among the reasons for catheter removal, the absence of records prevailed in $51.02 \%(\mathrm{n}=25)$, followed by obstruction and infection, both with $12.24 \%(\mathrm{n}=6)$. Regarding the length of stay, it was noted that of the 49 catheters implanted, $55.10 \%(\mathrm{n}=27)$ had no records and $36.74 \%(\mathrm{n}=18)$ remained for less than one year. Conclusion: The evidenced data reinforce the need for nursing records and the application of good practices for the maintenance and handling of these catheters. Emphasis is placed on the importance of training the nursing team in order to promote patient safety through qualified nursing care.
\end{abstract}

Keywords: Nursing; Adolescent health; Neoplasia; Hematology; Central venous catheterization.

\title{
Resumen
}

Objetivo: analizar el uso de catéteres venosos centrales de larga duración en adolescentes con enfermedades oncohematológicas, atendidos en el período comprendido entre 2008 y 2018, en una sala especializada de atención integral a la salud del adolescente. Método: Estudio de análisis documental, transversal, retrospectivo, con abordaje cuantitativo, desarrollado en un Hospital Universitario del Estado de Rio de Janeiro, Brasil. Resultados: Muestra compuesta por 49 formularios sobre las condiciones clínicas de adolescentes con enfermedades onco-hematológicas sometidos a la implantación de catéteres venosos centrales. El 65\% ( $\mathrm{n}=32)$ eran varones con una edad mínima y máxima de 12 y 19 años, con un 22,45\% $(\mathrm{n}=11)$ de 15 años. En cuanto a las enfermedades oncohematológicas, predominaron las leucemias, especialmente la leucemia linfoide aguda, representada en el $43 \%(\mathrm{n}=21)$ de los casos, seguida de los linfomas en el 35\% (n = 17). Entre los tipos de dispositivos, predominó el Catéter Central de Inserción Periférica en el 71,43\% ( $\mathrm{n}=35)$, y entre los motivos de retirada del catéter, predominó la ausencia de registros en el $51,02 \%(n=25)$, seguido de obstrucción e infección, ambas con 12,24\% $(n=6)$. En cuanto al tiempo de estancia, se observó que de los 49 catéteres implantados, el 55,10\% ( $\mathrm{n}=27)$ no tenían registros y el 36,74\% ( $\mathrm{n}=18)$ permanecieron menos de un año. Conclusión: Los datos evidenciados refuerzan la necesidad de registros de enfermería y la aplicación de buenas prácticas para el mantenimiento y manejo de estos catéteres. Se enfatiza la importancia de capacitar al equipo de enfermería para promover la seguridad del paciente a través de una atención de enfermería calificada.

Palabras clave: Enfermería; Salud de los adolescentes; Neoplasia; Hematología; Cateterismo venoso central.

\section{Introdução}

O presente estudo foi desenvolvido em uma enfermaria de atenção integral à saúde do adolescente, tendo por objeto a cateterização venosa central de longa permanência em adolescentes com doenças onco-hematológicas.

A adolescência é um período repleto de mudanças físicas, psicológicas, sociais, culturais, situado entre o final da infância e o início da vida adulta. Geralmente, é um tempo de intensas descobertas, muita vitalidade e energia (Eisenstein, 2005). No entanto, muitos adolescentes acabam adoecendo e necessitando de hospitalização em decorrência de diversas patologias e por causas externas, e dentre as doenças que acometem esta população destacam-se as neoplasias. Corrobora Borges et al (2009) 
ao ressaltar que a nível nacional as neoplasias malignas ocupam a terceira posição dentre as causas de óbito infanto-juvenil, sendo superadas apenas por mortes decorrentes de causas externas e acidentes, e compara o Brasil com os Estados Unidos, onde o câncer infantil também está entre as principais causas de mortalidade na faixa etária de 1 a 14 anos.

Em relação às neoplasias, as de origem hematológica são as que apresentam maior incidência em crianças e adolescentes (Brasil, 2015). Esse tipo caracteriza-se por alterações no sistema sanguíneo ou em hematopoiético, sendo conhecidas como leucemias, linfomas e síndromes mielodisplásicas. Ao se tratar da incidência do câncer infanto-juvenil, o Instituto Nacional de Câncer José Alencar Gomes da Silva (INCA) afirma que no Brasil a leucemia é o mais comum na maioria das populações (cerca de 25\% a 35\%) e os linfomas correspondem ao segundo tipo mais incidente no país (Brasil, 2015).

O INCA estima que em 2018, existam 10.800 novos casos de Leucemia na população brasileira, sendo o décimo tipo de câncer mais frequente na região Sudeste do Brasil. Em relação aos linfomas, estima-se que o não Hodgkin a incidência seja de 10.180 novos casos para cada ano do biênio 2018-2019, sendo a nível nacional o décimo primeiro mais incidente tanto para homens quanto para mulheres. Já o linfoma de Hodgkin tem uma estimativa de incidência de 2.530 casos para cada ano do biênio 2018-2019, esses dados correspondem a estimativa do risco de 1,43 casos novos a cada 100 mil homens, sendo a $14^{\text {a }}$ neoplasia mais frequente. No entanto, entre as mulheres, estima-se que o risco seja de 0,96 casos novos para cada 100 mil, ocupando a $17^{\mathrm{a}}$ posição (Brasil, 2017a).

$\mathrm{O}$ tratamento deste das neoplasias hematológicas baseiam-se na quimioterapia antineoplásica, tendo em vista que a via endovenosa a principal via de administração destas drogas. No entanto, os quimioterápicos podem causar toxicidades dermatológicas, nas quais dependendo do grau podem gerar o prolongamento do tempo de hospitalização e a interrupção temporária do tratamento. Dentre as toxicidades locais, destaca-se o extravasamento, considerado um dos principais eventos adversos, e que irá exigir do enfermeiro intervenções rápidas e tomadas de decisão. No que tange a prevenção recomenda-se o uso de um acesso venoso central para a administração segura deste tipo de droga. Portanto, a cateterização venosa central é procedimento amplamente utilizado na assistência à saúde em casos de pacientes que demandam certa complexidade no cuidado, principalmente para administração de medicações irritantes e vesicantes (Melo et al., 2020; Schneider \& Pedrolo, 2011; Santos et al., 2014).

Considera-se que o Cateter Venoso Central (CVC) é um sistema intravascular, que tem como principais inserções as veias: subclávia, jugular interna e femoral, sendo utilizadas para fluidoterapia, administração de fármacos, infusão de derivados sanguíneos, nutrição parenteral, monitorização hemodinâmica e a terapia renal substitutiva (Brasil, 2017b; Santos et al., 2014).

Ainda, em relação aos tipos de CVC, Ortolani, Gasparino e Traldi (2013) afirmam que eles são divididos conforme o tempo de permanência do dispositivo, relacionando com a terapia utilizada, podendo ser de longa permanência, utilizados em terapia endovenosa prolongada, ou de curta permanência, como os utilizados em unidades de terapia intensiva.

Neste sentido, o avanço tecnológico tem sido um importante aliado na luta contra o câncer, tanto na prevenção quanto no tratamento, pois dentre as principais inovações destacam-se os dispositivos intravasculares de uso prolongado, imprescindíveis para a terapêutica antineoplásica segura, além de serem capazes de promover conforto, aliviar a dor e proporcionar bem-estar ao paciente (Silva et al., 2009).

Os cateteres de longa permanência também possuem diferenças morfológicas, sendo divididos em semi-implantados ou totalmente implantados (port-a-cath), ambos possuem suas vantagens e desvantagens, como a minimização da ansiedade, por evitar repetidas punções, e maior segurança na administração de drogas vesicantes (Souza et al., 2013).

No que concerne aos cateteres de longa permanência, destaca-se ainda o cateter central de inserção periférica (CCIP) que se constituiu um excelente dispositivo a ser utilizado em pacientes de oncologia em tratamento antineoplásico. O CCIP possui menor custo-benefício, além de ser um dispositivo que não precisa de sala de cirurgia para a sua inserção (Bortoli et al., 2019). Reis et al (2019) destacam que a inserção do CCIP é um procedimento que pode ser realizado a beira leito, cabendo ao 
enfermeiro habilitado sua execução, conforme estabelecido pelo Conselho Federal de Enfermagem (COFEN) regulamentado através da Resolução n²58 de 2001 (Conselho Federal de Enfermagem, 2001).

No entanto, Santos et al. (2014) afirmam que apesar do uso desses dispositivos ser benéfico no tratamento de pacientes críticos, pode trazer riscos como tromboembolismo, infecções primárias da corrente sanguínea (IPCS), entre outros. Ainda, as infecções da corrente sanguínea (ICS) relacionadas a cateteres centrais (ICSRC) estão associadas a desfechos desfavoráveis em saúde (Brasil, 2017b). Considerando essas complicações e a clínica dos pacientes onco-hematológicos, ressalta-se a importância dos cuidados de enfermagem desde a inserção até a remoção desses dispositivos.

Além disso, reforça-se que a manipulação/manuseio de um cateter venoso central (CVC) é responsabilidade do enfermeiro e de sua equipe. Cabe a esse profissional garantir uma assistência de qualidade ao executar adequadamente a técnica de manuseio, evitando complicações como obstruções e infecções, através da identificação precoce de eventuais sinais de complicações (Silva et al., 2009). Soma-se a isso as ações de educação permanente no serviço, a construção e adesão a protocolos de cuidados padronizados baseados em evidências e o monitoramento dos indicadores assistenciais (Menezes, Bittencourt \& Menezes, 2013).

Em face do exposto, o presente estudo tem por objetivo analisar o uso dos cateteres venosos centrais, de longa permanência, em adolescentes com doenças onco-hematológicas, atendidos no período compreendido entre 2008 e 2018, em uma enfermaria especializada de atenção integral à saúde do adolescente.

\section{Metodologia}

Estudo descritivo transversal, retrospectivo realizado através de análise documental, com abordagem quantitativa sobre a implantação de cateter venoso central, de longa permanência, em pacientes onco-hematológicos, nos meses de janeiro e fevereiro de 2019, em uma enfermaria especializada de atenção integral a saúde do adolescente de um Hospital Universitário do Estado do Rio de Janeiro - RJ, Brasil. A pesquisa quantitativa é aquela em que se coletam e analisam dados quantitativos sobre variáveis diversas. Dessa forma, este tipo de pesquisa é capaz de identificar a natureza profunda das realidades, seu sistema de relações e sua estrutura dinâmica (Pereira et al., 2018).

A amostra constitui-se de 49 impressos do setor, nos quais estavam contidos registros de adolescentes com diagnóstico de doença onco-hematológica que foram submetidos ao procedimento cateterização venosa central de longa permanência, acompanhados desde a inserção até a retirada do cateter no período compreendido entre 2008 e 2018. Utilizou-se por critério de inclusão: impressos contendo dados de adolescentes de 12 a 18 anos que utilizaram o cateter venoso central de longa permanência para tratamento de doença onco-hematológica, incluindo o CCIP. Por critério de exclusão adotou-se: impressos contendo dados de adolescente de 12 a 18 anos que utilizaram cateter venoso central para tratamento de outras patologias e impressos que descreviam o uso de cateter venoso central de curta permanência.

As informações relativas à implantação e retirada, tanto à beira leito no caso do CCIP quanto cirúrgica no caso do totalmente e semi-implantados, o manuseio e a manutenção dos cateteres, são registrados pelos enfermeiros (as) e residentes de enfermagem capacitados/habilitados que atuam na unidade de internação especializada em saúde do adolescente.

Para fins deste estudo, elaborou-se um Formulário de Coleta de dados, com as seguintes variáveis a serem analisadas: sexo, idade, diagnóstico médico (conforme Classificação Internacional de Doenças-CID 10), indicação clínica do uso do cateter, tipo de cateter, tempo de permanência do cateter e o motivo de retirada.

A coleta de dados ocorreu no próprio setor, após autorização institucional. Durante esse processo, vale sinalizar, desde já, algumas limitações como a ausência de registros, principalmente em relação às variáveis "motivo de retirada e tempo de permanência".

Os dados coletados foram digitados e tabulados eletronicamente com auxílio do programa Microsoft Excel (2016). 
Aplicou-se estatística descritiva simples, com distribuição de frequências, relativa e absoluta.

Ressalte-se aqui, que foram respeitados todos os aspectos éticos, conforme Resolução $n^{\circ} 466$ de 2012 do Conselho Nacional de Saúde, e que este estudo foi aprovado no Comitê de Ética em Pesquisa sob o número de parecer 2.471.375 (Brasil, 2012). Por se tratar de uma pesquisa retrospectiva de análise documental de impressos próprios do setor, dispensou-se a assinatura do Termo de Consentimento Livre e Esclarecido.

\section{Resultados}

No presente estudo foram avaliados 49 impressos contendo informações sobre adolescentes submetidos a cateterização venosa central de longa permanência. Quanto a idade e sexo os dados estão apresentados na Tabela 1:

Tabela 1 - Distribuição de adolescentes submetidos à cateterização venosa central de longa permanência, conforme idade e sexo, em uma unidade especializada de um Hospital Universitário, de 2008 a 2018, Rio de Janeiro, RJ, Brasil, 2019 ( $n=49)$.

\begin{tabular}{ccccccccc}
\hline Idade & \multicolumn{2}{c}{ Feminino } & \multicolumn{2}{l}{ Masculino } & Não informado & \multicolumn{2}{c}{ Total } \\
\hline & $\mathbf{n}$ & $\mathbf{\%}$ & $\mathbf{n}$ & $\mathbf{\%}$ & $\mathbf{n}$ & $\mathbf{\%}$ & $\mathbf{n}$ & $\mathbf{\%}$ \\
12 anos & 1 & 2,04 & 5 & 10,20 & 0 & 0 & 6 & 12,24 \\
13 anos & 0 & 0 & 6 & 12,24 & 0 & 0 & 6 & 12,24 \\
14 anos & 4 & 8,16 & 2 & 4,08 & 0 & 0 & 6 & 12,24 \\
15 anos & 5 & 10,20 & 6 & 12,24 & 0 & 0 & 11 & 22,45 \\
16 anos & 4 & 8,16 & 1 & 2,04 & 0 & 0 & 5 & 10,20 \\
17 anos & 1 & 2,04 & 8 & 16,33 & 0 & 0 & 9 & 18,37 \\
18 anos & 0 & 0 & 3 & 6,12 & 0 & 0 & 3 & 6,12 \\
19 anos & 1 & 2,04 & 1 & 2,04 & 0 & 0 & 2 & 4,08 \\
Não informado & 0 & 0 & 0 & 0 & 1 & 2,04 & 1 & 2,04 \\
\hline Total & $\mathbf{1 6}$ & $\mathbf{3 2 , 6 5}$ & $\mathbf{3 2}$ & $\mathbf{6 5 , 3 1}$ & $\mathbf{1}$ & $\mathbf{2 , 0 4}$ & $\mathbf{4 9}$ & $\mathbf{1 0 0}$ \\
\hline
\end{tabular}

Fonte: Autores (2019).

Dos 49 impressos de adolescentes com doenças onco-hematológicas submetidos à implantação dos cateteres, 65\% $(\mathrm{n}=32)$ eram do sexo masculino. Quanto à faixa etária, a idade mínima e máxima eram respectivamente, 12 e 19 anos, sendo 22,45\% ( $\mathrm{n}=11$ ) adolescentes com a idade de 15 anos. Em relação aos dados não informados, quanto a idade e sexo, representam apenas $2 \%(\mathrm{n}=1)$ dos registros.

Ao mapear o tipo de doença onco-hematológica dos pacientes submetidos ao cateterismo venoso central de longa permanência, o Gráfico 1 aponta: 
Gráfico 1 - Distribuição de adolescentes submetidos ao cateterismo venoso central conforme diagnóstico médico, em uma unidade especializada de um Hospital Universitário, de 2008 à 2018, Rio de Janeiro, 2019 (n= 49).

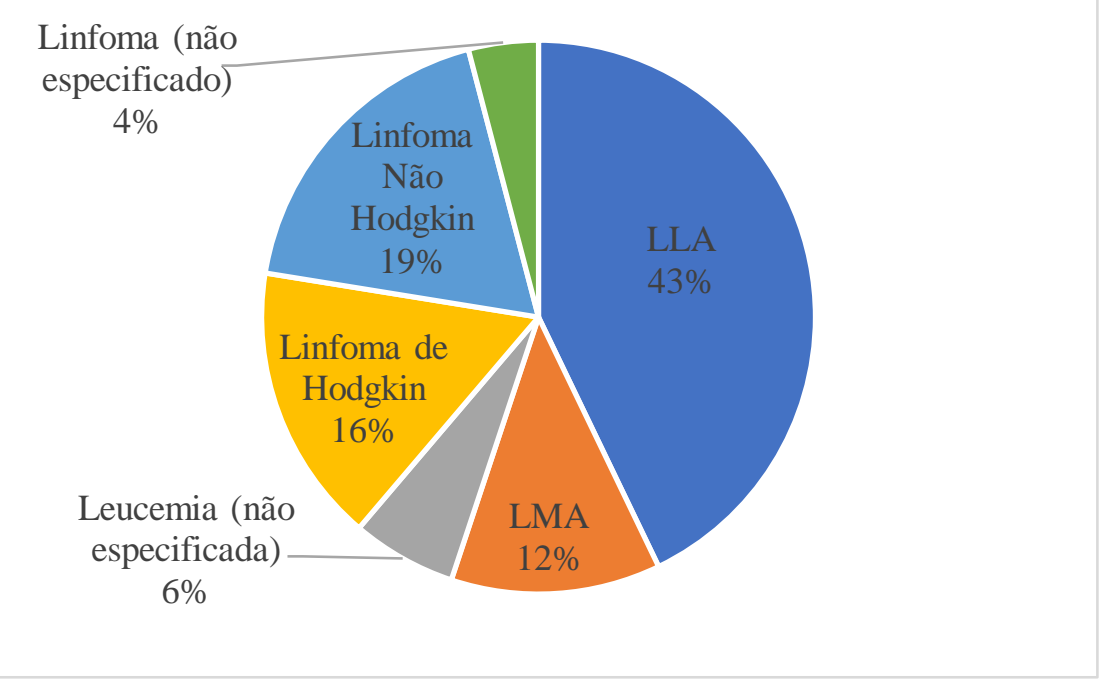

Fonte: Autores (2019).

No Gráfico 1, observou-se um predomínio das leucemias destacando-se a Leucemia linfoide aguda (LLA). representada por $43 \%(n=21)$ dos casos, seguida dos linfomas com 35\% (n=17). Quanto aos motivos de retirada segundo o tipo de cateter, a Tabela 2 apresenta:

Tabela 2 - Distribuição do motivo de retirada do cateter venoso central de longa permanência, segundo o tipo de cateter, em uma unidade especializada de um Hospital Universitário, de 2008 à 2018, Rio de Janeiro, 2019 $(n=49)$.

\begin{tabular}{|c|c|c|c|c|c|c|c|c|}
\hline \multirow[t]{2}{*}{ Motivo de Retirada } & \multicolumn{2}{|c|}{$\begin{array}{c}\text { Cateter } \\
\text { Totalmente } \\
\text { Implantado }\end{array}$} & \multicolumn{2}{|c|}{$\begin{array}{l}\text { Cateter Semi- } \\
\text { implantado }\end{array}$} & \multicolumn{2}{|c|}{ CCIP } & \multicolumn{2}{|c|}{ Total } \\
\hline & $\mathbf{n}$ & $\%$ & $\mathbf{n}$ & $\%$ & $\mathbf{n}$ & $\%$ & $\mathbf{N}$ & $\%$ \\
\hline Não Informado & 3 & 6,12 & 2 & 4,08 & 20 & 40,82 & 25 & 51,02 \\
\hline Infecção & 1 & 2,04 & 3 & 6,12 & 2 & 4,08 & 6 & 12,24 \\
\hline Obstrução & 0 & 0 & 0 & 0 & 6 & 12,24 & 6 & 12,24 \\
\hline Flebite & 0 & 0 & 0 & 0 & 2 & 4,08 & 2 & 4,08 \\
\hline Óbito & 2 & 4,08 & 0 & 0 & 0 & 0,0 & 2 & 4,08 \\
\hline Trombose & 0 & 0 & 0 & 0 & 2 & 4,08 & 2 & 4,08 \\
\hline Desposicionamento & 0 & 0 & 0 & 0 & 2 & 4,08 & 2 & 4,08 \\
\hline Fim de tratamento & 1 & 2,04 & 1 & 2,04 & 0 & 0,0 & 2 & 4,08 \\
\hline Troca & 1 & 2,04 & 0 & 0 & 0 & 0,0 & 1 & 2,04 \\
\hline Infiltração & 0 & 0 & 0 & 0 & 1 & 2,04 & 1 & 2,04 \\
\hline Total & 8 & 16,33 & 6 & 12,24 & 35 & 71,43 & 49 & 100 \\
\hline
\end{tabular}

Fonte: Autores (2019). 
Dentre os tipos dispositivos, foi possível perceber a predominância do CCIP, ilustrado por 71,43\% (n=35) dos cateterismos realizados na unidade. Concomitante a essa análise, destaca-se que dentre os motivos de retirada, prevaleceu a ausência de registros com 51,02\% (n=25), seguida da obstrução e da infecção, ambas com representação de 12,24\% (n=6).

Em relação à variável tempo de permanência, notou-se que dos 49 cateteres implantados, 55,10\% (n=27) apresentou ausência dos registros. Constatou-se que dentre as cateterizações, $36,74 \%(n=18)$ permaneceram em um tempo menor que um ano, conforme Tabela 3.

Tabela 3 - Distribuição do tempo de permanência de cateter venoso central de longa permanência, segundo o tipo de cateter, em uma unidade especializada de um Hospital Universitário, de 2008 a 2018, Rio de Janeiro, 2019 (n= 49).

\begin{tabular}{lll}
\hline Variável & $\mathbf{n}$ & $\mathbf{\%}$ \\
\hline Tempo de permanência & 18 & 36,74 \\
De 01 a 08 meses & 1 & 2,04 \\
De 09 a 16 meses & $\mathbf{n}$ & $\mathbf{\%}$ \\
\hline Tempo de permanência & 3 & 6,12 \\
De 17 a 24 meses & 27 & 55,10 \\
Não informado & $\mathbf{4 9}$ & $\mathbf{1 0 0}$ \\
\hline Total &
\end{tabular}

Fonte: Autores (2019).

\section{Discussão}

Em relação às doenças onco-hematológicas, a Leucemia Linfoide Aguda (LLA) foi a mais incidente entre os adolescentes submetidos ao cateterismo venoso central de longa permanência no período analisado. Este dado pode estar relacionado com fato de que as leucemias, de uma maneira geral, apresentam alta incidência e prevalência no público infantojuvenil, conforme demonstra o relatório do INCA, em que destaca a LLA, a nível mundial, como o câncer mais comum nessa população, tendo um percentual variando entre $25 \%$ e 35\%. (Brasil, 2017c).

No que se refere aos dados relacionados ao sexo, corroboram estudo realizado por Machado et al. (2017) que evidenciou que 59\% referem-se a adolescentes do sexo masculino. Neste sentido, Machado et al. (2017) afirmam que isso se se dá pelo fato de as leucemias serem mais prevalentes no sexo masculino, conforme as estimativas no Brasil (2018) que traz 5.940 casos novos de leucemias em homens e 4.860 em mulheres para cada ano do biênio 2018-2019.

A respeito da idade, nota-se que o presente estudo se encontra consonante com o estudo de Machado et al. (2017) onde houve a predominância de pacientes na faixa etária de 11 a 16 anos, assim como estudo desenvolvido por Presti et al. (2012) que evidenciaram que 59,6\% dos casos de adolescentes diagnosticados com câncer apresentavam faixa etária entre 10 e 14 anos, enquanto 40,4\% tinham entre 15 e 19 anos, sendo a idade média dos adolescentes de 13,8 anos.

Sabendo que se é necessário ter uma via segura de administração de quimioterápicos, foram analisados os cateteres de escolha da unidade, dentre eles o CCIP se destacou, seguido do Cateter Venoso Central Totalmente Implantado (CVC - TI). O CCIP é considerado um cateter venoso central de longa permanência, que propicia a atuação maior do profissional enfermeiro no ato de inserção. Evidenciando, uma característica particular da unidade, que valoriza a autonomia e atuação de profissionais enfermeiros, habilitados para inserir CCIP, concomitante com a tomada de decisão pelo melhor custo-benefício.

Estudo de Souza et al (2013) destacam que o cateter mais utilizado é o CVC-TI por ser considerado mais seguro. No entanto, no estudo o CVC-TI foi o segundo cateter mais inserido correspondendo a $16,33 \%$ ( $\mathrm{n}=8)$ dos cateteres inseridos na 
unidade estudada.

Ainda, em relação aos motivos de retirada, os dados corroboram com uma pesquisa realizada em um hospital universitário que identificou a ausência de registros como um fator limitante para a análise de tal variável (Machado et al., 2017). Reis et al. (2019) em pesquisa realiza em um serviço de atendimento integral a adolescentes, em relação ao CCIP, evidenciaram a falha de preenchimentos de algumas informações em documentos dos serviços, o que ocasiona prejuízo na precisão dos dados, principalmente nas variáveis tempo de permanência e motivo de retirada.

Quanto às complicações, Bortoli et al. (2019) destacam a infecção e a obstrução como as causas mais comuns de retiradas do CCIP. Ainda, aponta que portadores de Leucemia Mieloide Aguda (LMA) tinham mais chances de complicações infecciosas relacionadas ao seu uso. A infecção é considerada o problema mais grave relacionado ao uso de cateter de longa permanência, pois o tratamento antineoplásico tende a induzir imunossupressão, principalmente leucopenia, deixando o paciente extremamente suscetível a colonização por microrganismos oportunistas (Souza et al. 2013).

Este dado reforça a necessidade de educação permanente em serviço para adesão as boas práticas relacionadas à manutenção e ao manuseio dos cateteres venosos, bem como ao processo de implantação. O Manual de Medidas de Prevenção de Infecção Relacionada à Assistência à Saúde, da Agência Nacional de Vigilância Sanitária (ANVISA), aponta diversas instruções e orientações baseadas em estudos com evidência científica, sinalizando que a não prevenção das infecções de corrente sanguínea relacionadas a cateteres centrais (ICSRC) leva a complicações à saúde dos pacientes, como prolongamento das internações, agravamento do quadro clínico e aumento dos casos de óbito por sepse (Brasil, 2017b).

$\mathrm{Na}$ análise do tempo de permanência dos cateteres, observou-se a ausência de registros em 55,10\% ( $\mathrm{n}=27)$. No entanto, dos dados registrados, nota-se predomínio de um tempo de permanência menor que um ano. Por se tratar de um cateter de longa permanência presume-se um tempo de utilização prologando, haja vista, que este tipo de cateter é mais dispendioso economicamente. Estes dados dialogam com os resultados de estudo desenvolvido por Ortolani, Gasparino e Traldi (2013) em um ambulatório, no qual foram apontados um elevado número de dispositivos retirados com 10 meses de uso.

Os dados evidenciados reforçam a necessidade dos registros de enfermagem e da aplicação de boas práticas relacionadas à manutenção e manuseio destes cateteres. Ressalta-se ainda, a importância da capacitação da equipe de enfermagem quanto aos cuidados relacionados aos cateteres venosos de longa permanência, a fim de garantir a segurança do paciente por meio de uma assistência de enfermagem qualificada.

Dentre as limitações do estudo tem-se a constatação de importante déficit de registros de enfermagem, o que impede, em boa medida, uma análise com acurácia de dados relacionados ao perfil de cateterização venosa de longa permanência em adolescentes com doenças onco-hematológicas.

\section{Conclusão}

Ao analisar o uso de cateteres venosos centrais de longa permanência em adolescentes com doenças oncohematológicas, entre os anos de 2008 e 2018, o estudo evidenciou o predomínio de adolescente com LLA, em uso de CCIP, e dentre os motivos de retirada destacam-se as infecções e as obstruções; e o tempo de permanência inferior a um ano de uso.

Também foi possível observar, pelo estudo, escassez, ou quase inexistência de dados sobre as variáveis motivos de retirada e tempo de permanência dos cateteres. A escassez de tais dados, por sua vez, aponta a premente necessidade de melhorias no processo de registro da equipe de enfermagem.

Espera-se que os resultados alcançados venham contribuir para sensibilizar enfermeiros e equipe de enfermagem para a necessidade de se instituir programa de educação permanente da equipe sobre manuseio e manutenção dos principais cateteres de longa permanência utilizados na unidade. O conhecimento do perfil de implantação propicia a melhoria da qualidade do serviço, importante fator de impacto na qualidade de vida desses adolescentes. 
Com base nos mencionados resultados e limitações, sugere-se a realização de novos estudos sobre a temática, sobretudo, em relação ao tempo de permanência deste tipo de cateter, haja vista, que sua escolha deve levar em conta o período do tratamento. Por fim, recomenda-se a realização de estudos relacionados as complicações dos cateteres venosos centrais de longa permanência no adolescente portador de doenças onco-hematológicas, a fim de contribuir com as boas práticas de assistência de enfermagem e de saúde.

\section{Referências}

Borges, J. B. R., Loggetto, S., Giatti, M. J. L., de Camargo, A. C. M., Pereira, A. C.P., Miazaki, A. P., \& dos Santos, T. A. (2015, agosto). Caracterização das pacientes, na infância e adolescência, portadoras de câncer no município de Jundiaí e região. Revista Brasileira de Cancerologia. 55(4). 337-343. http://www1.inca.gov.br/rbc/n_55/v04/pdf/337_artigo3.pdf

Bortoli, P. S. de, Leite, Biaggi, A. C. A., Alvarenga, W. A., Alvarenga, C. S., Bessa, C. R., \& Nascimento, L. C. (2019, março). Cateter venoso central de inserção periférica em oncologia pediátrica: revisão de escopo. Acta paul. Enferm, $32(2)$. $220 \quad$ - 228. http://www.scielo.br/scielo.php?script=sci_arttext\&pid=S0103-21002019000200220\&lng=en\&nrm=iso

Brasil, Ministério da Saúde. (2015). Estimativa 2016: Incidência de câncer no Brasil. Instituto Nacional do Câncer José Alencar Gomes da Silva. http://www.inca.gov.br/bvscontrolecancer/publicacoes/edicao/Estimativa_2016.pdf.

Brasil, Ministério da Saúde. (2017a). Estimativa 2018: Incidência de Câncer no Brasil. Instituto Nacional do Câncer José Alencar Gomes da Silva. https://www.inca.gov.br/sites/ufu.sti.inca.local/files//media/document//estimativa-incidencia-de-cancer-no-brasil-2018.pdf.

Brasil, Agência Nacional de Vigilância Sanitária (2017b). Medidas de Prevenção de Infecção Relacionada à Assistência à Saúde. http://portal.anvisa.gov.br/documents/33852/3507912/Caderno+4+-+Medidas+de+Preven\%C3\%A7\%C3\%A3o+de+Infec\%C3\%A7\%C3\%A3o+Relaciona $\mathrm{da}+\% \mathrm{C} 3 \% \mathrm{~A} 0+$ Assist\%C3\%AAncia+\%C3\%A0+Sa\%C3\%BAde/a3f23dfb-2c54-4e64-881c-fccf9220c373.

Brasil, Ministério da Saúde. (2017c). Incidência, mortalidade e morbidade hospitalar por câncer em crianças, adolescentes e adultos jovens no Brasil. Resultados e comentários. Instituto Nacional do Câncer José Alencar Gomes da Silva. http://www1.inca.gov.br/wcm/incidencia/2017/pdf/versao-completa.pdf

Brasil, Conselho Nacional de Saúde. (2013, junho). Resolução n. 466, 2012. Diretrizes e Normas regulamentadoras de pesquisa envolvendo seres humanos. Seção 1 p. 59.

Resolução COFEN n. 258, de 12 de julho de 2001. Dispõe sobre a Inserção de Cateter Central Periférico, pelos enfermeiros. http://www.cofen.gov.br/resoluocofen-2582001_4296.html

Eisenstein, E. (2005). Adolescência: definições, conceitos e critérios. Revista Adolescência \& Saúde, 2(2), 6-7. https://cdn.publisher.gn1.link/a dolescenciaesaude.com/pdf/v2n2a02.pdf

Machado, L. B. L., Moura, D. A., Cunha, K. C. S., \& Cunha, L. B. C. (2017). Característica dos cateteres e de crianças portadoras de doença onco - hematológica. Cogitare Enfermagem, https://revistas.ufpr.br/cogitare/article/view/48448. http://dx.doi.org/10.5380/ce.v22il.48448

Melo, J. M. A., Oliveira, P. P. de, Souza, R. S., Fonseca, D., Gontijo, T. F., \& Rodrigues, A. B. (2020). Prevenção e conduta frente ao Extravasamento de agentes antineoplásicos: scoping review. Revista Brasileira de Enfermagem. https://www.scielo.br/scielo.php?script=sci_arttext \&pid= S003471672020000400303 \& tlng=en doi: https://doi.org/10.1590/0034-7167-2019-0008.

Menezes, V. P. S., Bittencourt, A. R., \& Menezes, M. F. B., (2013). Infecção relacionada a cateter venoso central: indicador de qualidade da assistência em oncologia. J. res fundam. Care online 5(3):373-385. http://www.seer.unirio.br/index.php/cuidadofundamental/article/viewFile/2303/pdf_888 10.9789/2175$5361.2013 v 5 n 3 p 373$.

Ortolani, L., Gasparino, R. C., \& Traldi, M. C. (2013). Complicações Associadas ao Uso de Cateter totalmente Implantável em Crianças e Adolescentes. Revista Brasileira de Cancerologia. 59(1), 51-56. http://www1.inca.gov.br/rbc/n_59/v01/pdf/08-complicacoes-associadas-ao-uso-de-cateter-totalmenteimplantavel.pdf

Pereira, A. S., Shitsuka, D. M., Parreira, F. J., \& Shitsuka, R. (2018). Metodologia da Pesquisa Científica. Santa Maria: universidade Federal de Santa Maria. P. 70. https://www.ufsm.br/app/uploads/sites/358/2019/02/Metodologia-da-Pesquisa-Cientifica_final.pdf

Presti, P. F., Macedo, C. R. D., Caran, E. M., Rodrigues, A. H. D., \& Petrilli, A. S. (2012). Estudo epidemiológico de câncer na adolescência em centro de referência. Revista Paulista de Pediatria. 30(2), 210-216. https://www.redalyc.org/pdf/4060/406038941009.pdf

Reis, N. S. P., Santos, M. F. G., Leite, D. C., Gomez, H. F., Peres, E. M., \& Junior, E. F. P. (2019). Implantação de cateter central de inserção periférica por enfermeiros em adolescentes. Cogitare Enfermagem. 24(55639). http://dx.doi.org/10.5380/ce.v24i0.55639

Santos, S.F., Viana, R. S., Alcoforado, C. L. G. C., Campos, C. C., Matos, S. S., \& Ercole, F. F. (2014). Ações de enfermagem na prevenção de infecções relacionadas ao cateter venoso central: uma revisão integrativa. Rev. SOBECC. 19(4), 219-225. https://doi.org/10.5327/Z1414-4425201400040008

Schneider, F., \& Pedrolo, E. (2011). Extravasamento de drogas antineoplásicas: avaliação do conhecimento da equipe de enfermagem. Revista Mineira de Enfermagem. 15(4), 522-529. https://cdn.publisher.gn1.link/reme.org.br/pdf/v15n4a08.pdf

Souza, G.S., Rocha, P. R. S., Reis, P. E. D., \& Vasques, C. I. (2013). Manuseio de cateter venoso central de longa permanência em pacientes portadores de câncer. Revista de Enfermagem do Centro Oeste Mineiro. 3(1), 577-586. http://www.seer.ufsj.edu.br/index.php/recom/article/viewFile/340/389

Silva, C. R. L. (2009) Acesso Vascular para Quimioterapia. Enfermagem Oncológica: Conceitos e Práticas. Yendis. 